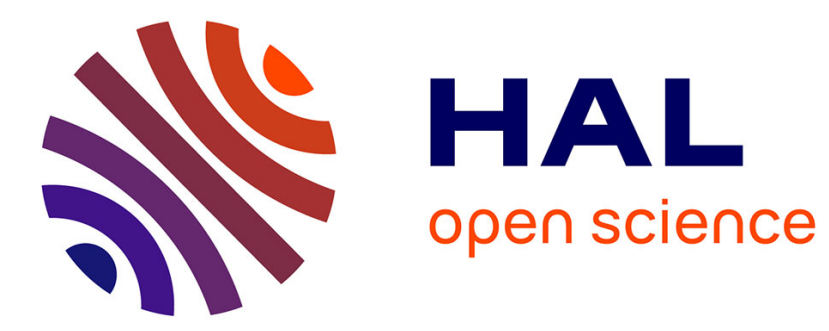

\title{
Lifetime of excitons in GaAs quantum wells
}

\author{
B. Sermage, S. Long, B. Deveaud, D. Katzer
}

\section{To cite this version:}

B. Sermage, S. Long, B. Deveaud, D. Katzer. Lifetime of excitons in GaAs quantum wells. Journal de Physique IV Proceedings, 1993, 03 (C5), pp.C5-19-C5-25. 10.1051/jp4:1993504 . jpa-00251502

\section{HAL Id: jpa-00251502 https://hal.science/jpa-00251502}

Submitted on 1 Jan 1993

HAL is a multi-disciplinary open access archive for the deposit and dissemination of scientific research documents, whether they are published or not. The documents may come from teaching and research institutions in France or abroad, or from public or private research centers.
L'archive ouverte pluridisciplinaire HAL, est destinée au dépôt et à la diffusion de documents scientifiques de niveau recherche, publiés ou non, émanant des établissements d'enseignement et de recherche français ou étrangers, des laboratoires publics ou privés. 


\title{
Lifetime of excitons in GaAs quantum wells
}

\author{
B. SERMAGE, S. LONG, B. DEVEAUD* and D.S. KATZER ${ }^{* *}$ \\ France Telecom, CNET, PAB, 196 avenue Henri Ravera, 92220 Bagneux, France \\ * France Telecom, CNET, LAB, 22300 Lannion, France \\ ${ }^{* *}$ Naval Research Laboratory, Washington, DC 20375, U.S.A.
}

\begin{abstract}
Time resolved luminescence measurements under resonant excitation were performed at $10 \mathrm{~K}$ on the free exciton in very high quality GaAs-AlAs quantum wells. At resonance, the luminescence intensity is increased by two orders of magnitude, and the decay is short. The radiative lifetime of the excitons in the radiant states is about $18 \mathrm{ps}$ in agreement with the theory of Hanamura and Andréani.
\end{abstract}

\section{INTRODUCTION}

Excitons in quantum wells are involved in new promising devices such as electro-optic modulators [1] or optical switching [2]. For this reason, the physical properties of the excitons in quantum wells have met new interest and particularly their radiative lifetime.

Many time resolved luminescence measurements on excitons at low temperature in GaAs-GaAlAs quantum wells have been reported till now. Some were performed with an excitation energy higher than the band gap [3], but in this case, the lifetime is perturbed by the cooling time of the excitons. However the results are that at $5 \mathrm{~K}$, the exciton lifetime vary between 0.3 and $1.6 \mathrm{~ns}$ when the well thickness is varied between 3 and $15 \mathrm{~nm}$ and that the lifetime increases strongly with temperature.

Time resolved luminescence measurements were also performed under resonant excitation [4-8]. Though there is some ambiguity between resonant Rayleigh scattering and resonant luminescence in the interpretation of the experiments, the main results are that at low excitation, the radiative lifetime of the excitons is very short [6,7] and that the lifetime increases with the excitation density [8]. In this paper we present new results concerning the time resolved spectral width, the polarization of the luminescence, the delay between the laser pulse and the luminescence and the influence of the excitation power on the luminescence decay.

\section{EXPERIMENTAL PROCEDURE}

We have made time resolved luminescence measurements on a very high quality MBE grown sample. The sample contains a $5.8 \mathrm{~nm}$ thick Gäss well between two $4.8 \mathrm{~nm}$ thick AlAs barriers [9]. It was grown with growth interruption at the GaAs-AlAs interfaces to minimize the interface roughness so that the width of the luminescence exciton peak at low temperature is $1.2 \mathrm{meV}$ and that the peaks due to the different numbers of monolayers are perfectly distinct [9]. During the experiments, the sample was maintained at 10 $\mathrm{K}$ and was excited by the laser pulses of a Titanium-Saphire cw mode locked laser. The pulses were $1.5 \mathrm{ps}$ long and their repetition rate was $82 \mathrm{MHz}$. The luminescence is observed in back scattering geometry and the sample is tilted so that the reflected beam does not go to the detector. The luminescence is spectrally dispersed by a $32 \mathrm{~cm}$ monochromator and temporally dispersed by a syncroscan streak camera. The monochromator is equipped with a 1200 groves $/ \mathrm{mm}$ grating which is mostly masked except $4 \mathrm{~mm}$ in the central region so that the temporal dispersion is smaller than our $10 \mathrm{ps}$ time resolution. The spectral resolution is $0.8 \mathrm{meV}$. On each sample, we excite first out of resonance and we choose a place on the sample where 
the excitonic luminescence spectrum is very narrow in focus mode (without time dispersion), where the scattered light is very weak and where the $n+1$ and $n-1$ monolayers luminescence peaks are very weak. Then we move the laser wavelength to the resonance i.e. to the maximum of the luminescence intensity and we observe that there is no Stokes shift between the luminescence energy and the resonance energy.

\section{RESULTS}

\section{1. Low excitation}

We observe at low excitation power $\left(-0.1 \mathrm{~W} / \mathrm{cm}^{2}\right)$ a strong increase of the luminescence intensity $(1000 \mathrm{x})$
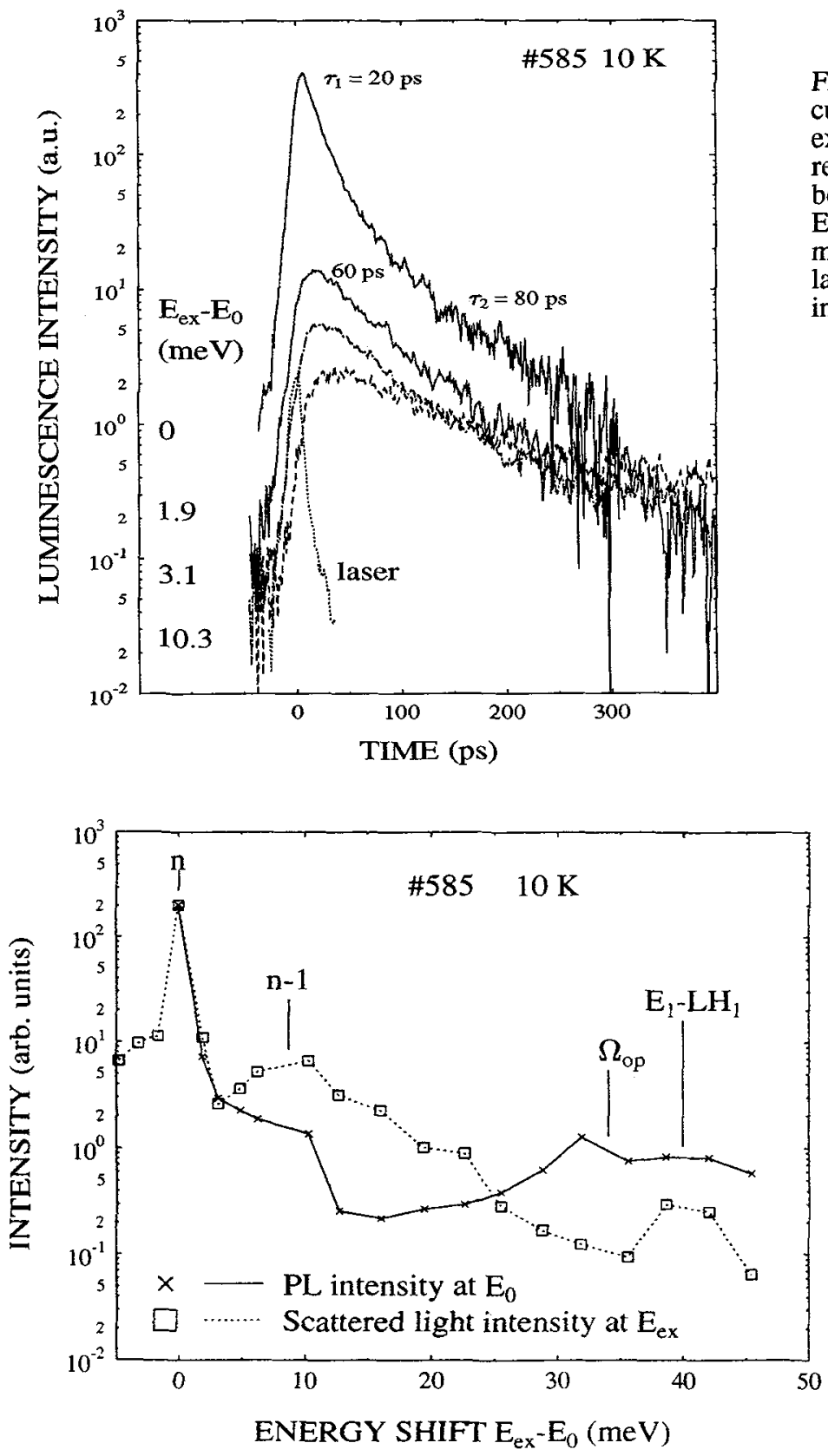

Fig. 1. Luminescence decay curves obtained for different excitation energy $E_{e x}$ above the resonance $\mathrm{E}_{0}$. From the top to the bottom, the curves correspond to $\mathrm{E}_{\mathrm{ex}}-\mathrm{E}_{0}=0 ., 1.9,3.1$ and 10.3 meV. The scattered light of the laser out of resonance is shown in dotted line.
Fig. 2. Variation of the luminescence intensity at the energy $E_{0}$ (solid line) and of the scattered light intensity at the energy $\mathrm{E}_{\mathrm{ex}}$ (dotted line) with the energy shift $E_{e x}-E_{0} . n$ and n-1 are the energies of the heavy hole exciton for $n$ and $n-1$ atomic monolayers, $\Omega_{p}$ is the surface phonon energy and $E_{1}-\mathrm{LH}_{1}$ is the light hole exciton for n monolayers. 
and a decrease of the decay time when the sample is excited at resonance (Fig. 1,2).

The figure 1 shows the luminescence decay curves at $E_{0}$ for different values of the excitation energy $E_{e x}$. The figure 2 shows the variation of the luminescence intensity (at the energy $E_{0}$ ) and of the intensity of the scattered light (at the energy $E_{e x}$ ) as a function of the detuning $E_{e x}-E_{0}$. In addition to the main resonance at $E_{0}$, we observe smaller resonances at about $E_{0}+8.8 \mathrm{meV}$ for $\mathrm{n}-1$ monolayers, at about $E_{0}+40 \mathrm{meV}$ due to the light hole exciton.

At resonance, the emission is the sum of two exponentials: a first one with a decay time $\tau_{1} \sim 20$ ps and a second one with a decay time $\tau_{2} \sim 80 \mathrm{ps}$. As we can see on figure 1 , the first decay $\tau_{1}$ is not limited by our time resolution since the decay time of the scattered light of the laser out of resonance is 3 ps. The figure 3 shows the time resolved luminescence in polarization parallel and perpendicular to that of the laser, the difference $I_{1-}-I_{\perp}$ and the spectral width at half maximum of the luminescence.

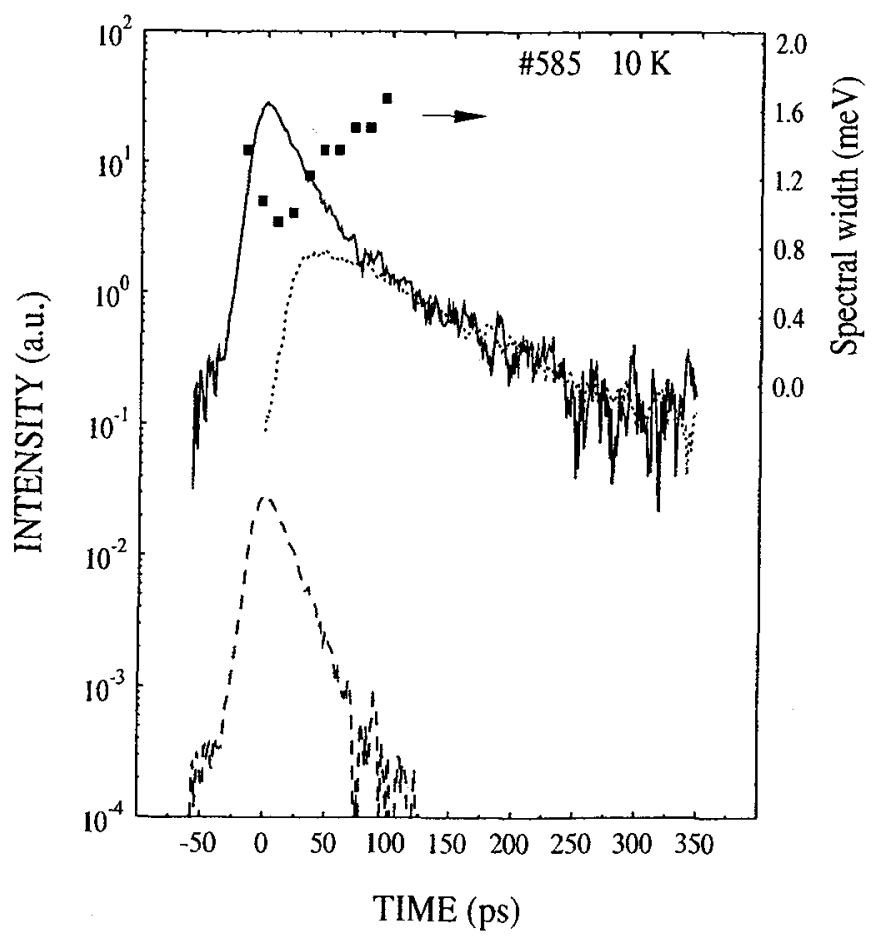

Fig. 3. Luminescence decay curves in polarization parallel (full line) and perpendicular (dotted line) to that of the excitation pulse. Decay of the difference between these intensities (dashed line). The full squares are the spectral width at half maximum.

The first signal (decay time $\tau_{1}$ ) is polarized parallel to the laser pulse and the spectral width is small $(\sim 1$ $\mathrm{meV}$ ) and close to the resolution of our apparatus. Same kind of experiments have been reported recently $[4,5,8,10]$ and the first signal was interpreted as resonant Rayleigh scattering [11]. We think as ref. [12] that this first signal is luminescence for the following reasons: $i)$ When the excitation energy is $3 \mathrm{meV}$ above the resonance (as in fig. 1), the scattering of the laser and the luminescence are well separated spectrally and yet the decay time at the beginning of the luminescence decay is 60 ps which is smaller than the second decay time at resonance which is $80 \mathrm{ps}$; ii) There is a delay of about 10 ps between the laser pulse and the luminescence peak at resonance as shown on the figure 4. Indeed we have reported on this figure the decay curves at resonance obtained successively at high excitation $\left(70 \mathrm{~W} / \mathrm{cm}^{2}\right)$ and at low excitation $\left(3.5 \mathrm{~W} / \mathrm{cm}^{2}\right)$. We have reproduced many times this experiment and we always observe that the low excitation luminescence is delayed by about $10 \mathrm{ps}$ compared to the high excitation one. The two different excitation powers are obtained by replacing a high density glass filter by a low density one of same thickness so that the time delay due to the filters is the same at high excitation and at low excitation. At high excitation, as we will see later, the ratio between the luminescence intensity and the excitation power $I_{I} / P_{e x}$ is reduced by more than one order of magnitude and the Rayleigh scattering on the surface of the sample can become larger than the resonant luminescence which is the case here. The first signal at high excitation corresponds to the laser pulse and the luminescence at low excitation is delayed relative to the laser pulse by about $10 \mathrm{ps}$. 


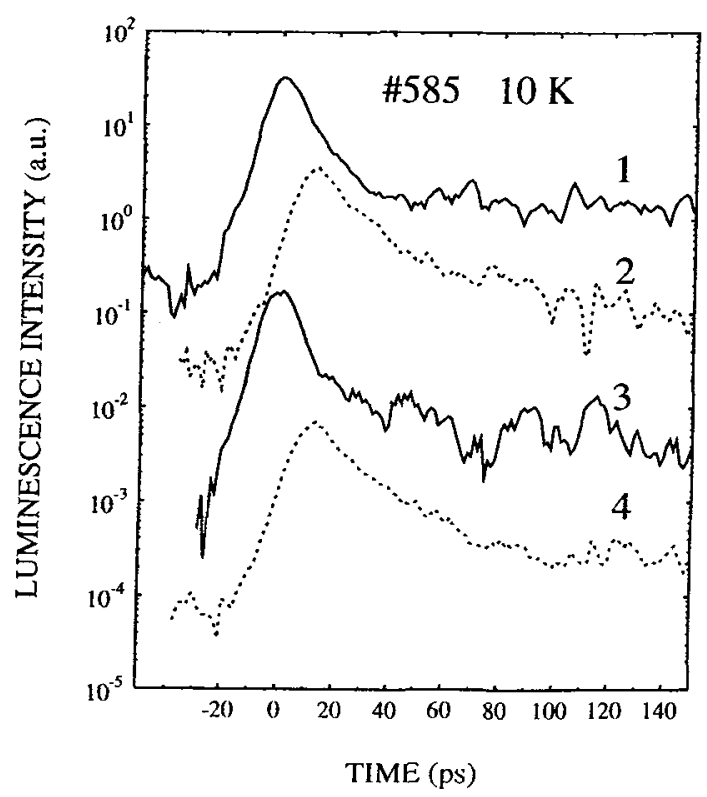

Fig. 4. Luminescence decay curves with resonant excitation obtained successively in this order: $1,2,3,4$. There is about 5 minutes delay between two acquisitions. The solid curves correspond to an excitation density of 70 $\mathrm{mW} / \mathrm{cm}^{2}$ and the dotted curves to 3.5 $\mathrm{mW} / \mathrm{cm}^{2}$. The curves have been displaced vertically. The two curves at high excitation are maximum at the same time which corresponds to the laser pulse. This shows that there is no jitter. The two low excitation curves are delayed by about 10 ps relative to the high excitation ones.

We thus consider that during the excitation pulse, there is creation of excitons in the quantum well with $k_{11}<\omega / c$. These excitons (in state " $1 "$ ) can not be observed in our experiment since they radiate in the direction of the reflected beam. They can either be scattered (with the characteristic time $\tau_{s}$ ) by the interface roughness to states "2" with $\mathrm{k}_{11}<\bar{n} \omega / \mathrm{c}$ (where $\bar{n}$ is the refractive index of the material) called radiant states where they can recombine with the lifetime $\tau_{0}$. This adiabatic scattering keeps the polarization of the excitons because the longitudinal-transverse splitting is nearly 0 at small $\mathrm{k}$ [13]. They can also be scattered (with the characteristic time $\tau^{\prime \prime}$ to "non radiant" states with $\mathrm{k}_{\| 1}>\bar{n} \omega / \mathrm{c}$ or $\mathrm{J}=2$ where they cannot recombine. The number of excitons in state 1 is $n_{1}$, in state 2 is $n_{2}$ and the number of thermalized excitons is $n_{3}$. The luminescence intensity $I_{L}$ is given by:

$$
I_{L} \sim \frac{n_{2}}{\tau_{0}}+\frac{n_{3}}{\tau_{t h}}
$$

where $\tau_{t h}$ is the radiative lifetime of the thermalized excitons. The first signal rises with the time $\tau_{s}^{\prime}$ and decreases with the time constant $\tau_{1}$ given by:

$$
\frac{1}{\tau_{1}}=\frac{1}{\tau_{0}}+\frac{1}{\tau_{s}^{\prime \prime}}
$$

The spectral width of this luminescence $\delta \mathrm{E}_{\mathrm{h}}$ is given by the following equation [10]:

$$
\delta E_{h}=2 \hbar\left(\frac{1}{2 \tau_{1}}+\frac{1}{\tau_{s}^{\prime}}\right) \text {. }
$$

The second signal with the decay time $\tau_{c h}$ is unpolarized. It corresponds to the recombination of thermalized excitons. The decay time $\tau_{t h}$ is longer because only the excitons which are in the radiant states can recombine [13]:

$$
\tau_{t h}=\tau_{0} \frac{n_{2}}{n_{3}}
$$


The spectral width of the luminescence is larger for the second signal ( $\delta \mathrm{E} \sim 1.4 \mathrm{meV}$ ) on one hand because the excitons diffuse to places which have other resonant energies $\left(\Gamma_{\text {in }}\right)$ and on the other hand because of the increase of the homogenous broadening $\Gamma_{h}$ due to the fact that the excitons are uncorrelated and have larger $\mathrm{k}$ [14].

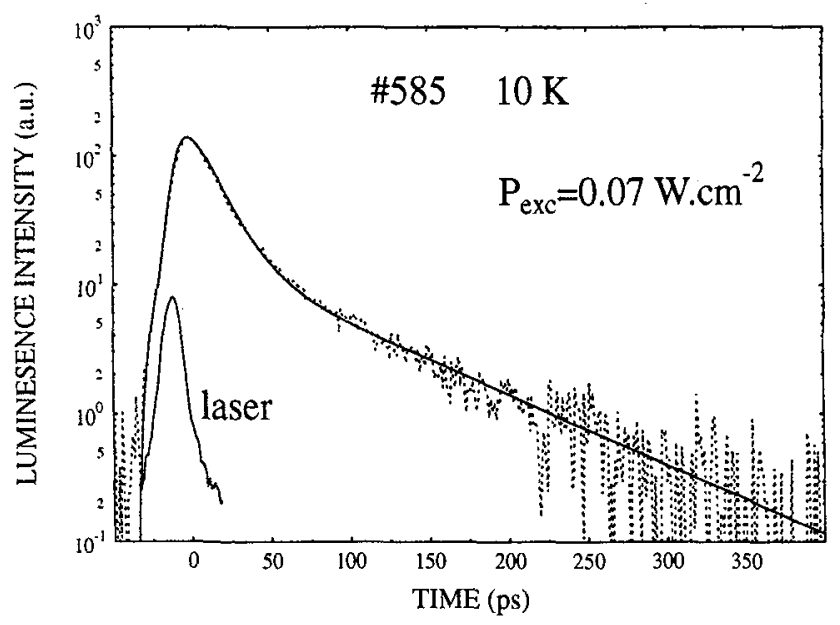

Fig. 5. Luminescence decay curve at 10 $\mathrm{K}$ at resonance with $0.07 \mathrm{~W} / \mathrm{cm}^{2}$ excitation power density (dashed line). The solid line is a calculated curve with $\tau_{s}^{\prime}$ $=5 \mathrm{ps}, \tau_{0}=18 \mathrm{ps}, \tau_{s}^{\prime \prime}=75 \mathrm{ps}$ and $\tau_{t h}=$ $80 \mathrm{ps}$ and the generation function for the excitons shown on the figure as "laser". There is about 11 ps delay between the laser pulse and the luminescence maximum.

The fit of the experimental curve obtained at $0.07 \mathrm{~W} / \mathrm{cm}^{2}$ with our model is given on the figure 5 . The calculated curve corresponds to the following values of the parameters: $\tau_{s}{ }_{s}=5 \mathrm{ps}, \tau_{0}=18 \mathrm{ps}, \tau^{\prime \prime}{ }_{s}=75 \mathrm{ps}$ and $\tau_{t h}=80$ ps. Andreani [13] has calculated the lifetime of the thermalized excitons:

$$
\tau_{t h}=\frac{3 M k_{B} T}{\hbar^{2} k_{0}^{2}} \tau_{0}
$$

where $M$ is the translation mass of the exciton, $k_{B}$ is the Boltzman constant and $k_{0}=\bar{n} \omega / c$ is the photon wave vector. This equation gives $\tau_{t h} / \tau_{0}=13.6$ at $10 \mathrm{~K}$ with an exciton mass $M=0.25 \mathrm{~m}_{0}$. In our case, we obtain $\tau_{z h} / \tau_{0}=4.4$. The discrepancy is problably due to the fact that the excitons are not well thermalized after 200 ps. The experimental value of $\tau_{t h}$ cannot be due to a tunnel of the excitons through the $4.8 \mathrm{~nm}$ thick AlAs barriers. Indeed we have calculated that the tunnel time (in the resonant case) is 7 ps for the electrons and $20 \mathrm{~ns}$ for the holes which makes the tunnel time for the excitons much larger than $\tau_{t h}$. Finally we find for this sample that the radiative lifetime of the excitons in the radiant states $\tau_{0}$ is about $18 \mathrm{ps.}$

\section{2. Influence of the excitation power}

We have made time resolved luminescence with resonant excitation on the same sample for different values of the excitation power between 0.04 and $70 \mathrm{~W} / \mathrm{cm}^{2}$. Some decay curves are shown on figure 6 and spectra on figure 7 .

When the excitation power increases, the first decay time $\tau_{1}$ becomes shorter and is limited by the resolution of our apparatus. This is due to the shortening of the exciton - exciton scattering time $\tau^{\prime \prime}{ }_{s}$. The intensity of the first signal increases sublinearly with the excitation power. This can be due to a lowering of the absorption coefficient at resonance due to the increase of the homogeneous broadening or to the increase of the radiative lifetime $\tau_{0}$. On the other hand, the decay time of the thermalized luminescence $\tau_{t h}$ get longer. As shown in ref. [8], this could be due to the increase of the homogeneous broadening which increases with the exciton concentration as it can be observed on the luminescence spectra of the figure 7 . However the variation of $\tau_{i n}$ with the excitation power do not agree with the expression given in ref. 8 taking for the homogeneous 
broadening $\Gamma_{h}(\mathrm{meV})=0.1+0.16610^{-10} n_{e x}$ as in ref. [15]. We think that the variation of $\tau_{t h}$ with the excitation density is not well understood up to now.

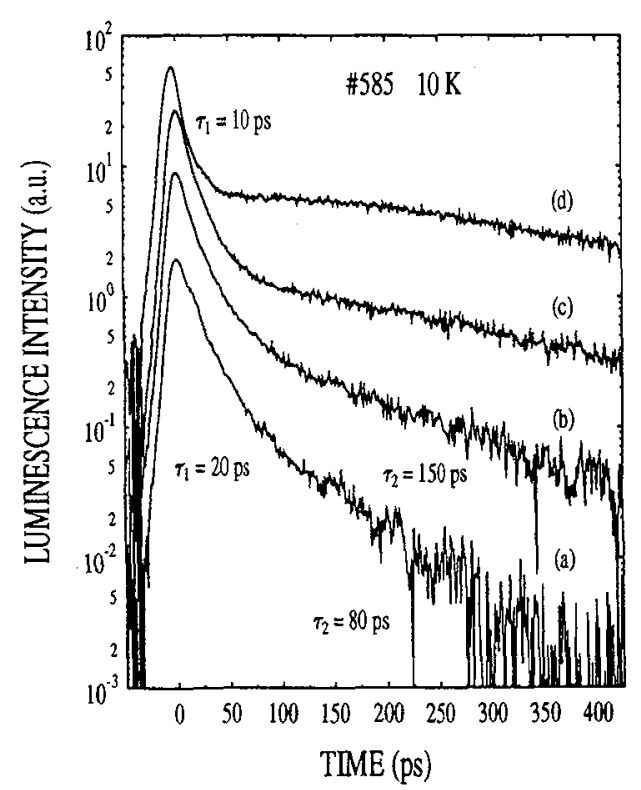

Fig. 6. Luminescence decay curves at resonance for different excitation power. From the bottom to the top, the excitation density power is: $0.07,0.56,2.2,35 \mathrm{~W} / \mathrm{cm} 2$.

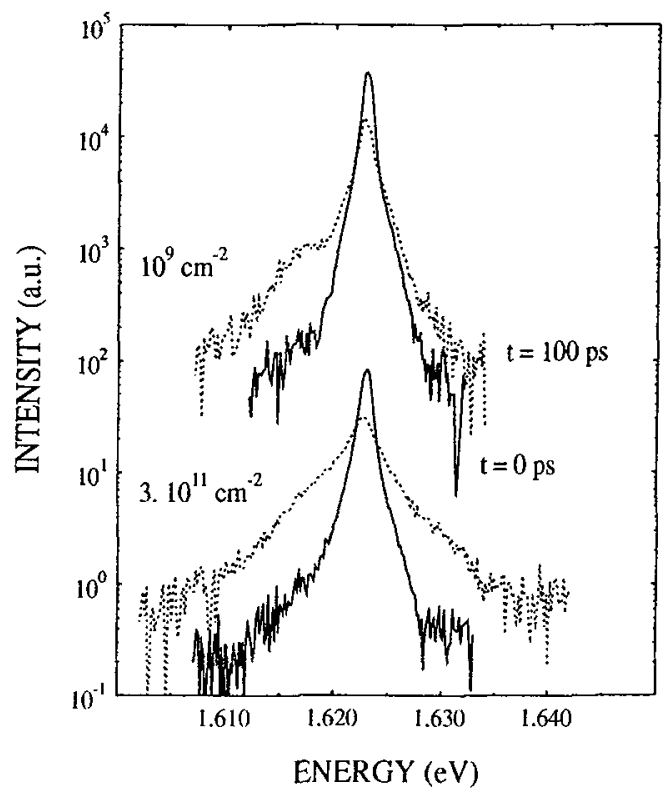

Fig. 7. luminescence spectra at the beginning (full line) and at the end (dotted line) of the luminescence decay. The upper curves correspond to an excitation power density of $0.07 \mathrm{~W} / \mathrm{cm}^{2}$. The lower curves are for 35 $\mathrm{W} / \mathrm{cm}^{2}$.

\section{CONCLUSION}

We have shown that the radiative recombination lifetime of the free excitons in high quality GaAs-AlAs $5.6 \mathrm{~nm}$ thick quantum wells is about $18 \mathrm{ps}$ for the excitons in the radiant states $(\mathrm{J}=1, \mathrm{k}<\bar{n} \omega / \mathrm{c})$, and $80 \mathrm{ps}$ for the thermalized excitons at low excitation at $10 \mathrm{~K}$. The value of $\tau_{i h}$ depends strongly on the excitation power.

\section{ACKNOWLEDGEMENTS}

We would like to thank I. Abram, C. Tanguy, B. Jusserand, J. Worlock and J. L. Oudar for helpful discussions. We are very debtfull to R. Raj for the loan of a streak camera.

\section{REFERENCES}

[1] Wood T.H., IEEE J. Lightwave Technol., LT-6 (6) (1988) 743.

[2] Miller D.A.B., Chemla D.S., Damen T.C., Gossard A.C., Wiegman W., Wood T.H. and Burrus C.A., Appl. Phys. Lett. 45 (1984) 13-15.

[3] Feldman J., Peter G., Göbel E.O., Dawson P., Moore K., Foxon C., and Elliot R.J., Phys. Rev. Lett. 59 (1987) 2337-2340.

[4] Oberhauser D., Kalt H., Schlapp W., Nickel H. and Klingshirn C., Journal of Luminescence 48 \& 49 (1991) 717-720.

[5] Stolz H., Schwarze D., von der Osten W. and Weiman G., Superlattices and Microstructures 9 (1991) 511-515. 
[6] Deveaud B., Clerot F., Roy N., Satzke K., Sermage B. and Katzer D.S., Phys. Rev. Lett. 67 (1991) 2355-2358.

[7] Sermage B., Deveaud B., Satzke K., Clérot F., Dumas C., Roy N., Katzer D.S., Mollot F., Planel R., Berz M. and Oudar J.L., Superlattices and Microstructures 13 (1993) 271-273.

[8] Eccleston R., Feuerbacher B.F., Kuhl J., Rühle W.W., and Ploog K., Phys. Rev. B 45 (1992) 11403-11406.

[9] Gammon D., Shanabrook B.V. and Katzer D.S., Appl. Phys. Lett. 57 (1990) 2710-2712.

[10] Stolz H., Schwarze D., von der Osten W. and Weimann G., Phys. Rev. B 47 (1993) 9669-9675.

[11] Hegarty J., Sturge M.D., Weisbuch C., Gossard A.C. and Wiegmann W., Phys. Rev. Lett. 49 (1982).

[12] Damen T.C., Leo K., Shah J. and Cunningham J.E., Appl. Phys. Lett. 58 (1991) 1902-1904.

[13] Andreani L.C., Solid State Comm. 77 (1991) 641-645.

[14] Oberhauser D., Pantke K.H., Hvam J.M., Weimann G. and Klingshirn C., Phys. Rev. B 47 (1993) 6827-6830.

[15] Honold A., Schultheis L., Kuhl J. and Tu C.W., Phys. Rev. B 40 (1989) 6442-6445. 